\title{
Interaction onde électromagnétique/flux de porteurs dans une structure mixte ferrite-semiconducteur
}

\author{
M. Baribaud et D. Rauly \\ Laboratoire d'Electromagnétisme, Circuits micro-ondes, E.R.A. 1014, E.N.S.E.R.G. \\ 23, rue des Martyrs, 38031 Grenoble Cedex, France
}

(Reçu le 19 avril 1984, accepté le 12 octobre 1984)

\begin{abstract}
Résumé. - L'interaction cumulative d'une onde électromagnétique avec un flux de porteurs a été étudiée dans une structure mixte ferrite-semiconducteur : en milieu solide, l'onde se propage dans la lame de ferrite alors que les porteurs sont entraînés dans le semiconducteur. Une première étude, qualitative, du mécanisme de l'interaction onde-porteurs détermine les possibilités de l'amplification. La deuxième partie, à l'aide du critère de Briggs, permet de préciser le comportement du dispositif et les conditions optimales de fonctionnement dans une structure de dimensions infinies. Dans le cas de dimensions finies, un comportement d'amplificateur sélectif en bande $\mathrm{X}$ est envisageable.
\end{abstract}

\begin{abstract}
Electromagnetic wave-carrier wave interaction has been studied in a ferrite-semiconductor structure : in solid medium, the electromagnetic wave is propagated in the ferrite and the carriers were drift in the semiconductor. In the first part a qualitative study of the wave-carrier interaction determines the amplification possibilities. The second part, from the Briggs instability criterium, states precisely the device behaviour and the optimal working conditions in infinite dimension structure. In the finite dimension case, a selective $\mathrm{X}$ band amplifier behaviour can be considered.
\end{abstract}

\section{Introduction.}

L'interaction cumulative d'une onde électromagnétique avec un flux de porteurs a été étudiée par de nombreux auteurs [1-3]. Elle a d'ailleurs servi de base théorique pour l'étude du comportement du tube à onde progressive [4]. En milieu solide, l'onde se propage dans une lame de ferrite alors que les porteurs sont entraînés dans un semiconducteur. Nous avons donc étudié l'interaction entre une onde électromagnétique se propageant dans un ferrite YIG et un flux de porteurs circulant dans un semiconducteur InSb adjacent [5]. Devant les difficultés rencontrées pour exprimer cette interaction sous la forme d'un couplage entre l'onde électromagnétique et une onde de charge d'espace comme dans un tube à onde progressive, nous avons été conduits à rechercher les zones d'instabilités à l'aide du critère de Briggs [6]. Les résultats obtenus précisent le comportement d'une structure YIG-InSb de dimensions infinies.

\section{Présentation du système.}

1.1 GÉOMÉTRIE DE LA STRUCTURE. - On considère une structure mixte semi-infinie constituée d'un ferrite et d'un semiconducteur séparés par le plan yoz comme le montre la figure 1 . Le ferrite correspond aux valeurs de $x<0$ et le semiconducteur aux valeurs de $x>0$. L'induction magnétique statique $\mathbf{B}_{0}$ est dirigée suivant l'axe $o z$ et la propagation s'effectue perpendiculairement à $\mathbf{B}_{0}$ suivant $o y$. Comme le modèle est infini

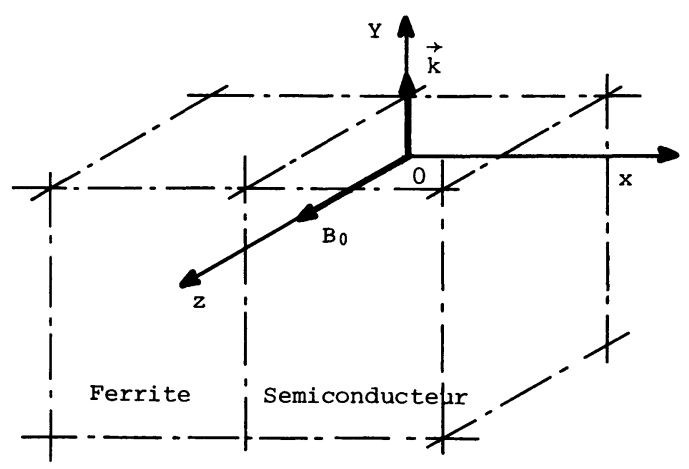

Fig. 1. - Géométrie de la structure et orientations du vecteur d'induction magnétique $\mathbf{B}_{0}$ et du vecteur d'onde $\mathbf{k}$.

[Structure geometry and magnetic induction vector $\mathbf{B}_{0}$ and wave vector $\mathbf{k}$ orientations.] 
dans la direction $o z$, les champs électromagnétiques ne varient pas suivant cette direction et s'écrivent :

Dans le ferrite $(x<0): \mathbf{X}_{m}=\mathbf{X}_{0 m} \mathrm{e}^{j\left(\omega t-k y-\gamma_{m} x\right)}$.

Dans le semiconducteur $(x>0)$ :

$$
\mathbf{X}_{\mathrm{s}}=\mathbf{X}_{0 \mathrm{~s}} \mathrm{e}^{j\left(\omega t-k y-\gamma_{\mathrm{s}} x\right)} .
$$

1.2 MODES PROPRES DANS LA STRUCTURE SEMI-INFINIE. - Les modes propres dans la structure mixte ferrite-semiconducteur sont des modes $\operatorname{TE}\left(E_{z}, H_{x}, H_{y}\right)$ et $\operatorname{TM}\left(H_{z}, E_{x}, E_{y}\right)$ [5].

Comme nous le verrons lorsque nous analyserons son mécanisme, pour qu'il existe une interaction entre l'onde électromagnétique et le flux des porteurs, il faut que la vitesse de phase de l'onde soit de l'ordre de grandeur de la vitesse des porteurs dans le semiconducteur, ce qui mène à des nombres d'onde voisins de $10^{5} \mathrm{~m}^{-1}$. Ceci conduit de nombreux auteurs $[2,7,8]$ à faire l'approximation magnétostatique qui consiste à supposer que $\operatorname{rot} \mathbf{H} \simeq 0$.

Bien que nous n'appliquions pas cette approximation dans notre étude, elle reste valable pour évaluer l'ordre de grandeur de l'amplitude des champs électromagnétiques du mode TM. Ces champs s'écrivent :

a) Dans le milieu magnétique :

$$
\mathbf{H}_{\mathrm{m}}=\left|\begin{array}{c}
0 \\
0 \\
A
\end{array}\right| \quad \mathbf{E}_{\mathrm{m}}=\left|\begin{array}{c}
E_{x \mathrm{~m}} \\
E_{y} \\
0
\end{array}\right| .
$$

b) Dans le milieu semiconducteur :

$$
\mathbf{H}_{\mathrm{s}}=\left|\begin{array}{c}
0 \\
0 \\
A
\end{array}\right| \quad \mathbf{E}_{\mathrm{s}}=\left|\begin{array}{c}
E_{x \mathrm{~s}} \\
E_{y} \\
0
\end{array}\right| .
$$

Les expressions (1) et (2) ont été écrites en tenant compte de la continuité des composantes tangentielles des champs à l'interface $x=0$. Les composantes $E_{x \mathrm{~m}}$, $E_{x s}$, et $E_{y}$ sont toutes proportionnelles à l'amplitude $A$ du champ magnétique [5]. Compte tenu des équations (1) et (2), l'équation rot $(\mathbf{H}) \simeq \mathbf{0}$ admet comme solution unique :

$$
\begin{gathered}
\left|\mathbf{H}_{\mathrm{m}}\right|=\left|\mathbf{H}_{\mathrm{s}}\right| \simeq 0 \\
\left|\mathbf{E}_{\mathrm{m}}\right| \simeq 0 \quad\left|\mathbf{E}_{\mathrm{s}}\right| \simeq 0 .
\end{gathered}
$$

Les modes TM ont donc une amplitude négligeable et seuls les modes TE offrent un intérêt pour obtenir des interactions entre l'onde et le flux de porteurs dans cette structure.

2. Etude des modes TE dans une structure semi-infinie.

2.1. EquATION DE DISPERSION. - On va étudier complètement le mode TE qui existe simultanément dans le ferrite et le semiconducteur en introduisant la relation de continuité des composantes tangen- tielles des champs électromagnétiques à la surface de séparation des deux milieux. On pose :

$$
n=k C / \omega ; \quad m=\gamma_{\mathrm{m}} C / \omega ; \quad s=\gamma_{\mathrm{s}} C / \omega .
$$

2.1.1 Propagation dans le ferrite. - Un ferrite soumis à l'action d'un champ magnétique statique parallèle à l'axe $o z$ peut être considéré comme un milieu à permittivité scalaire $\varepsilon_{0} \varepsilon_{\mathrm{r}}$ et à perméabilité tensorielle $\mu_{0} \overrightarrow{\vec{\mu}}_{\mathrm{m}}$ telle que :

$$
\overrightarrow{\vec{\mu}}_{\mathrm{m}}=\left|\begin{array}{ccc}
\mu_{1} & j \mu_{2} & 0 \\
-j \mu_{2} & \mu_{1}^{\prime} & 0 \\
0 & 0 & \mu_{3}
\end{array}\right| .
$$

Lorsque l'anisotropie magnétocristalline est négligeable, on a $\mu_{1}=\mu_{1}^{\prime}$. Dans ces conditions, on a [2] :

$$
\mu_{1}=\mu_{1}^{\prime}=1+\frac{\omega_{\mathrm{m}} \omega_{\mathrm{r}}}{\omega_{\mathrm{r}}^{2}-\omega^{2}}
$$

et

$$
\mu_{2}=\frac{\omega_{\mathrm{m}}}{\omega_{\mathrm{r}}^{2}-\omega^{2}}
$$

avec :

$\omega:$ pulsation du signal.

$\omega_{\mathrm{m}}=\gamma \boldsymbol{M}_{\mathrm{s}} ; \gamma$ étant le rapport gyromagnétique et $\boldsymbol{M}_{\mathrm{s}}$ l'aimantation à saturation du ferrite.

$\omega_{\mathrm{r}}=\gamma H_{0}+\omega_{\text {ech }} a^{2} k^{2}-j v_{\mathrm{m}} ; H_{0}$ étant le champ magnétique statique, $\omega_{\mathrm{ech}} a^{2} k^{2}$ un terme qui rend compte des effets d'échange d'énergie entre les spins, $v_{\mathrm{m}}$ la pulsation de relaxation (elle représente les pertes magnétiques).

L'équation de dispersion relative au mode TE s'écrit [5] :

$$
n^{2}+m^{2}=\varepsilon_{\mathrm{r}} \frac{\mu_{1}^{2}-\mu_{2}^{2}}{\mu_{1}} .
$$

2.1.2 Propagation dans le semiconducteur. - Un matériau semiconducteur soumis à l'action d'un champ magnétique statique parallèle à l'axe oz peut être considéré comme un milieu à perméabilité scalaire $\mu_{0}$ et à perméabilité tensorielle $\varepsilon_{0} \overrightarrow{\vec{\varepsilon}}_{\mathrm{s}}$ telle que [8] :

où

$$
\begin{aligned}
\overrightarrow{\vec{\varepsilon}}_{\mathrm{s}}=\left|\begin{array}{ccc}
\varepsilon_{1} & j \varepsilon_{2} & 0 \\
-j \varepsilon_{2} & \varepsilon_{1} & 0 \\
0 & 0 & \varepsilon_{3}
\end{array}\right| \\
\varepsilon_{1}=\varepsilon_{\mathrm{s}}-\frac{\omega_{\mathrm{p}}^{2}\left(\omega-k v_{0}-j v_{\mathrm{e}}\right)}{\omega\left[\left(\omega-k v_{0}-j v_{\mathrm{e}}\right)^{2}-\omega_{\mathrm{c}}^{2}\right]} \\
\varepsilon_{2}=\frac{\omega_{\mathrm{p}}^{2} \omega_{\mathrm{c}}}{\omega\left[\left(\omega-k v_{0}-j v_{\mathrm{e}}\right)^{2}-\omega_{\mathrm{c}}^{2}\right]} \\
\varepsilon_{3}=\varepsilon_{\mathrm{s}}-\frac{\omega_{\mathrm{p}}^{2}\left(\omega-k v_{0}\right)}{\omega^{2}\left(\omega-k v_{0}-j v_{\mathrm{e}}\right)}
\end{aligned}
$$


avec :

$\omega_{\mathrm{p}}=\left(N e^{2} / M \varepsilon_{0}\right)^{1 / 2}=v_{\mathrm{e}} \sigma_{0} / \varepsilon_{0}:$ pulsation plasma,

$\omega_{\mathrm{c}}=e B_{0} / M$ : pulsation cyclotronique.

$v_{\mathrm{e}}$ est la pulsation de collision, $\sigma_{0}$ la conductivité statique du semiconducteur en absence de champ magnétique statique, $-e$ la charge de l'électron, $M$ sa masse effective et $N$ la densité de porteurs majoritaires dans la bande de conduction. $B_{0}=\mu_{0} H_{0}$ est l'induction magnétique statique qui règne dans le semiconducteur.

$\varepsilon_{\mathrm{s}}$ est la permittivité relative scalaire du semiconducteur.

L'équation de dispersion relative au mode TE s'écrit [5] :

$$
n^{2}+s^{2}=\varepsilon_{3} \text {. }
$$

2.1.3 Relation de continuité. - La configuration des champs électromagnétiques à l'abscisse $x=0$ est représentée sur la figure 2 .

Les composantes tangentielles des champs $\mathbf{E}$ et $\mathbf{H}$ sont continues à la surface de séparation des deux milieux, ce qui donne la relation de continuité suivante :

$$
m-\frac{\mu_{1}^{2}-\mu_{2}^{2}}{\mu_{1}} s=j \frac{\mu_{2}}{\mu_{1}} n .
$$

La résolution du système d'équations $(6,11,12)$ conduit à une équation du $4^{\mathrm{e}}$ degré en $n=k C / \omega$, ce qui donne quatre racines pour le mode TE, représentées par leur nombre d'onde $k_{i}=k_{i}^{\prime}+j k_{i}^{\prime \prime}$ $(i=1,2,3,4)[5]$.

Les variations des parties réelles $k_{i}^{\prime}$ et imaginaires $k_{i}^{\prime \prime}$ en fonction de la fréquence sont représentées sur les figures 3 et 4 dans le cas d'une structure YIG-InSb plongée dans une induction magnétique statique de 0,4 tesla.

Les grandeurs caractéristiques du ferrite YIG sont les suivantes [9] :

- l'aimantation à saturation : $\mu_{0} M_{\mathrm{s}}=0,182 \mathrm{~T}$;

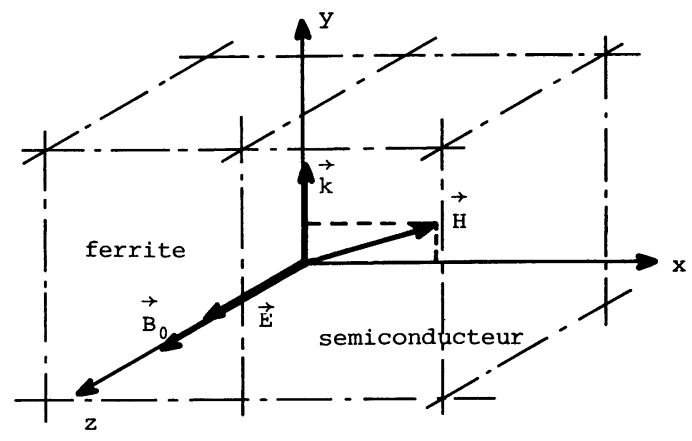

Fig. 2. Champ électromagnétique en $x=0$ pour le mode TE dans la structure mixte.

[TE electromagnetic field mode at $x=0$ in the structure.]

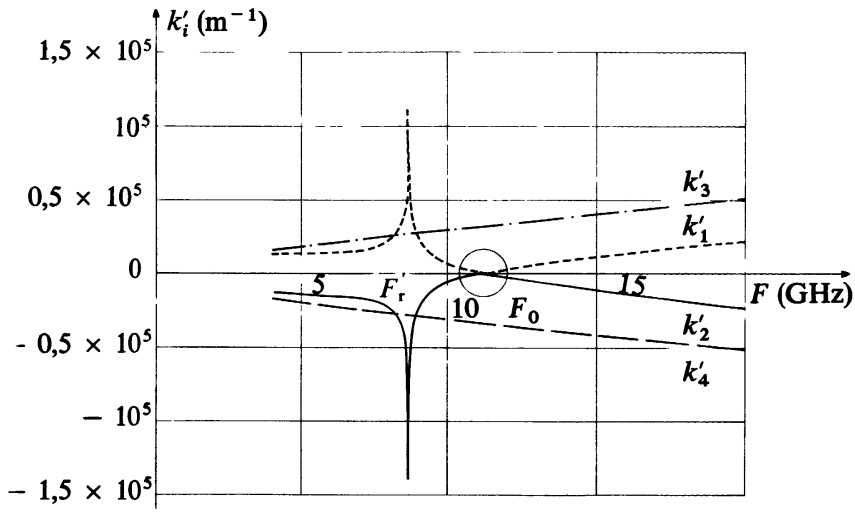

Fig. 3a. - Variations des parties réelles $k_{i}^{\prime}$ en fonction de la fréquence. Sur cette figure, l'identification des racines $k_{1}^{\prime}$ et $k_{2}^{\prime}$ n'est pas aisée, notamment dans la zone encerclée, centrée sur la fréquence $F_{0}=\omega_{0} / 2 \pi=\gamma B_{0} / 2 \pi$. Ces deux racines présentent un maximum pour la fréquence de résonance magnétique dans le ferrite :

$$
F_{\mathrm{r}}=\frac{\omega_{\mathrm{r}}}{2 \pi}=\frac{\gamma}{2 \pi}\left(B_{0}-\frac{\mu_{0} M_{\mathrm{s}}}{2}\right) .
$$

[ $k_{i}^{\prime}$ real part versus frequency. Here the $k_{1}^{\prime}$ and $k_{2}^{\prime}$ root identification is not easy, namely in circled zone around $F_{0}=\omega_{0} / 2 \pi=\gamma B_{0} / 2 \pi$. Both roots exhibit a top at the magnetic resonance frequency in ferrite : $F_{\mathrm{r}}=\omega_{\mathrm{r}} / 2 \pi=$ $\gamma\left(B_{0}-\mu_{0} M_{\mathrm{s}} / 2\right) / 2 \pi$.]

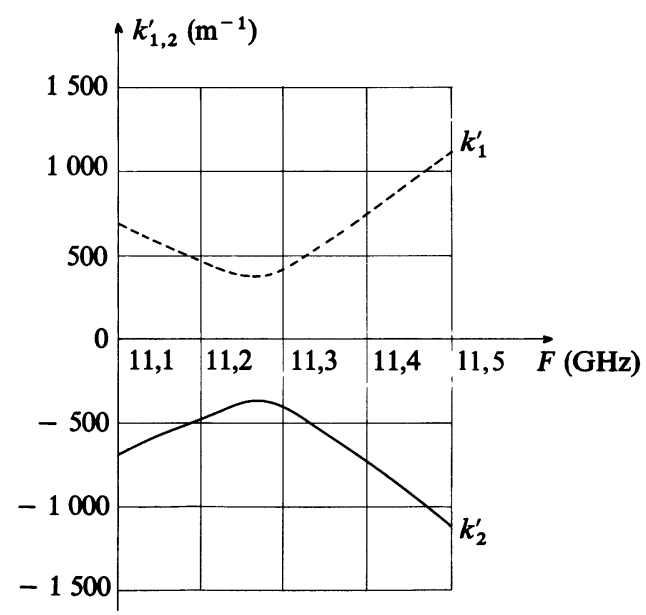

Fig. 3b. - Agrandissement au voisinage de $F_{0}(11,3 \mathrm{GHz})$. Aucun doute n'existe plus quant à l'identité des racines $k_{1}^{\prime}$ et $k_{2}^{\prime}$.

[Enlargement around $F_{0}(11.3 \mathrm{GHz})$. No doubt subsists about $k_{1}^{\prime}$ and $k_{2}^{\prime}$ identities.]

- la pulsation d'amortissement :

$$
v_{\mathrm{m}}=2,8 \times 10^{7} \mathrm{rad} / \mathrm{s} ;
$$

- la permittivité relative : $\varepsilon_{\mathrm{r}}=14,5$. 


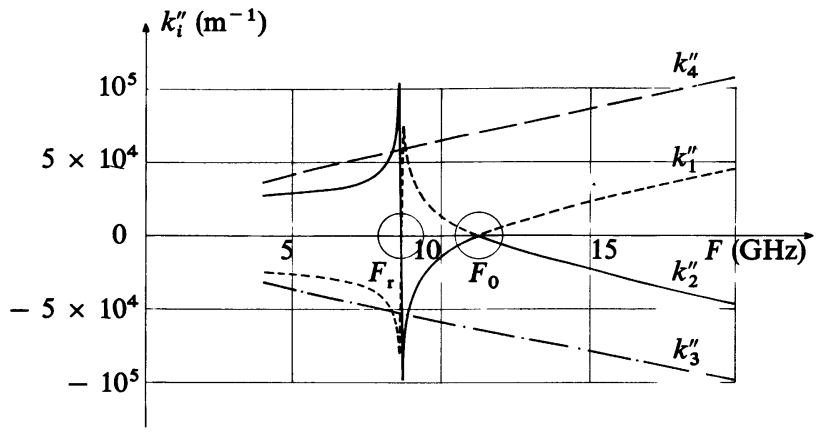

Fig. 4a. - Variations des parties imaginaires $k_{i}^{\prime \prime}$ en fonction de la fréquence. Les zones encerclées centrées sur $F_{0}$ et $F_{\mathrm{r}}$ posent des difficultés pour l'identification des racines $k_{1}^{\prime \prime}$ et $k_{2}^{\prime \prime}$. Ces deux racines s'annulent au voisinage de $F_{0}$ et $F_{\mathrm{r}}$. Cette ambiguité est levée par les figures $4 b$ et $4 c$.

$\left[k_{i}^{\prime \prime}\right.$ imaginary parts versus frequency. The circled zones around $F_{0}$ and $F_{\mathrm{r}}$ put some difficulties for $k_{1}^{\prime \prime}$ and $k_{2}^{\prime \prime}$ root identification. Both roots are zero around $F_{0}$ and $F_{\mathrm{r}}$. The ambiguity is solved in figures $4 b$ and $4 c$.

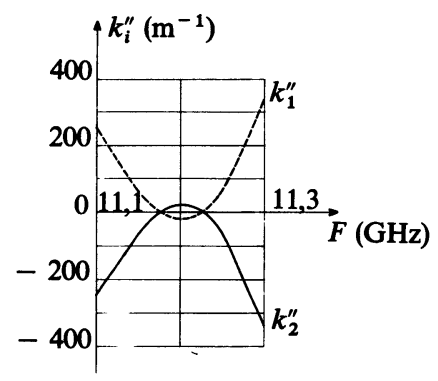

Fig. 4b. - Agrandissement au voisinage de $F_{0}(11,3 \mathrm{GHz})$. [Enlargement near $F_{0}(11.3 \mathrm{GHz})$.]

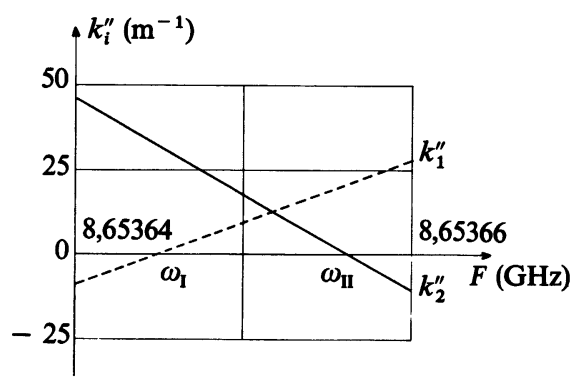

Fig. 4c. - Agrandissement au voisinage de $F_{\mathrm{r}}(8,65365 \mathrm{GHz})$.

[Enlargement near $F_{\mathrm{r}}(8.65365 \mathrm{GHz})$.]

Les grandeurs caractéristiques du semiconducteur InSb sont les suivantes [10] :

- la conductivité statique : $\sigma_{0}=10^{4} \Omega^{-1} \mathrm{~m}^{-1}$;

- la pulsation de collision :

$$
v_{\mathrm{e}}=2 \pi \times 1500 \times 10^{9} \mathrm{rad} / \mathrm{s} ;
$$

- la permittivité relative scalaire $: \varepsilon_{\mathrm{s}}=18,7$.
La vitesse d'entraînement des porteurs dans le semiconducteur est de $10^{5} \mathrm{~m} / \mathrm{s}$.

\subsection{STRUCTURE DES MOdES TE.}

2.2.1 Structure des modes 1 et 2 . - Les figures 3 et 4 montrent que les modes 1 et 2 présentent des singularités aux pulsations $\omega_{0}=\gamma B_{0}$ et $\omega_{\mathrm{r}}=\gamma\left(B_{0}-\frac{\mu_{0} M_{\mathrm{s}}}{2}\right)$. $\left(\omega_{\mathrm{r}}\right.$ est la pulsation de résonance ferromagnétique, compte tenu des champs démagnétisants dans le ferrite.)

On peut montrer que, au voisinage de $\omega_{\mathrm{r}}$, les composantes transversales du vecteur d'onde de ces modes s'écrivent :

$\gamma_{\mathrm{m}_{1}}=j k_{10} \quad$ et $\quad \gamma_{\mathrm{s}_{1}}=-j k_{10}$

( $k_{10}$ nombre réel positif)

et

$\gamma_{\mathrm{m}_{2}}=-j k_{20} \quad$ et $\quad \gamma_{\mathrm{s}_{2}}=j k_{20}$

( $k_{20}$ nombre réel positif).

Les champs électromagnétiques des deux modes décroissent exponentiellement en s'éloignant de l'interface $x=0$. On trouve des valeurs faibles pour la vitesse de phase, à savoir :

$$
V_{\phi_{1}}=5,3 \times 10^{5} \mathrm{~m} / \mathrm{s} \text { et } V_{\phi_{2}}=-4,2 \times 10^{5} \mathrm{~m} / \mathrm{s} \text {. }
$$

Donc, au voisinage de la pulsation de résonance $\omega_{\mathrm{r}}$, les deux modes possèdent une structure d'onde lente de surface se propageant dans des directions opposées.

Lorsqu'on s'éloigne de la pulsation $\omega_{\mathrm{r}}$, ces deux modes reprennent une structure d'onde de volume avec la particularité qu'aucune propagation n'existe pour $\omega=\omega_{0}$ puisque $k_{1} \simeq k_{2} \simeq 0$.

2.2.2 Structure des modes 3 et 4 . - $\mathrm{Si} B_{0}$ était inférieur à $\mu_{0} M_{\mathrm{s}} / 2$, les modes 3 et 4 présenteraient une singularité à la pulsation $\omega_{\mathrm{r}}^{\prime}=\gamma\left(\mu_{0} \boldsymbol{M}_{\mathrm{s}} / 2-\boldsymbol{B}_{0}\right)$. Ce n'est pas notre cas comme on peut le constater sur les figures 3 et 4.

Ces deux modes gardent une vitesse de phase et de groupe sensiblement constantes sur toute la gamme de fréquence étudiée. Ils ont une structure d'onde de volume.

2.3 IMPORTANCE DES PHÉNOMÈNES DE COUPLAGE. Les modes TE que nous venons d'analyser sont ceux qui existent dans une structure YIG-InSb en cas de couplage entre l'onde électromagnétique et le flux de porteurs dans le semiconducteur. Lorsque ce couplage n'existe pas, on obtient quand même une propagation d'ondes lentes de surface qui se manifeste également sous la forme de quatre modes TE [5]. Ce résultat correspond avec ceux de nombreux auteurs [11, 12]. Ces ondes existent aussi dans des lames de ferrite seules $[7,13]$. En résumé, le couplage introduit par l'interaction de l'onde électromagnétique avec le flux de porteurs ne modifie pas fondamentalement la structure de cette onde. Cependant, il est nécessaire d'étudier la nature et le mécanisme de cette interaction afin d'en préciser les effets. 


\section{Mécanisme de l'interaction onde-porteurs.}

3.1 ACTION DU FLUX DE PORTEURS DANS LE SEMICONDUCTEUR SUR L'AIMANTATION DANS LE FERRITE. L'interaction de l'onde électromagnétique dans le ferrite avec le flux de porteurs dans le semiconducteur s'explique à l'aide des figures 5 et 6 .

Sur la figure 5, le flux de porteurs est représenté par un courant statique $I_{0}$ dirigé suivant oy. Le champ magnétique $\mathbf{H}$ de l'onde et le champ d'induction $\mathbf{B}=\overrightarrow{\vec{\mu}} \mathbf{H}$ sont tous deux dans le même plan xoy.

Par effet Hall, l'action de la composante $B_{x}$ sur le courant $I_{0}$ fait apparaître des courants $\mathbf{i}$ de même fréquence que le signal, dirigés sur l'axe oz et qui créent des champs hyperfréquences modifiant la précession des spins dans le ferrite.

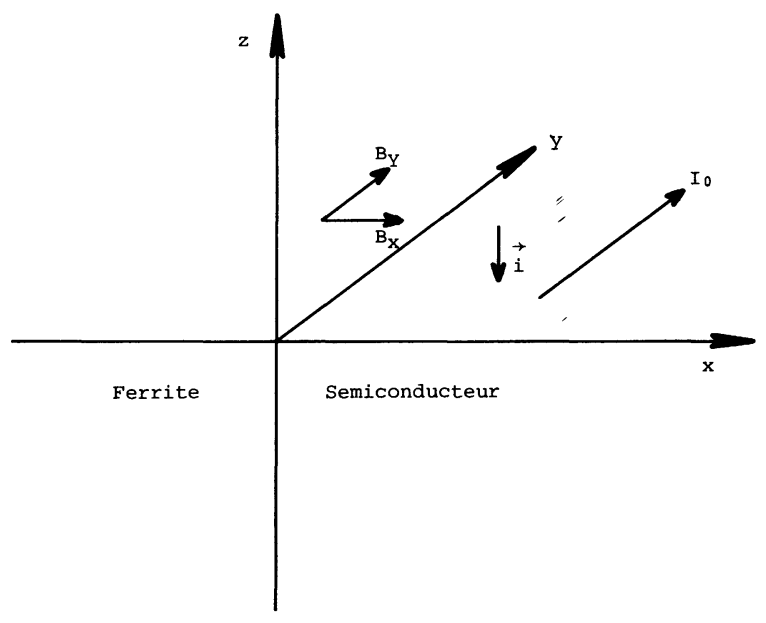

Fig. 5. - Orientation des courants mis en jeu et du champ d'induction magnétique hyperfréquence.

[Current and microwave magnetic field orientations.]

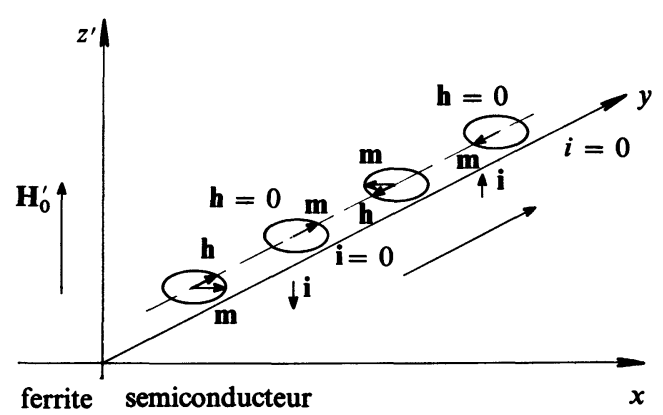

Fig. 6. - Positions de la composante dynamique $\mathbf{m}$ de l'aimantation dans le ferrite, du courant de Hall $\mathbf{i}$ dans le semiconducteur et du champ h créé par i. L'écart entre deux positions correspond à un quart de la longueur d'onde guidée.

[Magnetization dynamic component $\mathbf{m}$ in the ferrite, Hall current $\mathbf{i}$ in the semiconductor and created-by-i field $\mathbf{h}$ locations. The difference between both positions corresponds to a quarter of the wave length.]
L'aimantation $\mathbf{M}$ (macroscopique) dans le ferrite résulte de la composante statique $\mathbf{M}_{\mathrm{s}}$ dirigée suivant $\mathbf{o z}$ et de la composante hyperfréquence $\mathbf{m}$ située dans le plan xoy. La composante $M_{x}$ est en avance d'un quart de période sur $M_{y}$, ce qui donne à $\mathbf{M}$ un mouvement de précession $[1,7]$. La figure 6 représente la position de $\mathbf{m}$ et du courant $\mathbf{i}$ en plusieurs points séparés d'un quart de la longueur d'onde guidée à la fréquence de résonance ferromagnétique. Le champ magnétique hyperfréquence $h$, créé par le courant $i$ est dirigé suivant l'axe oy, il est en avance d'un quart de période sur l'aimantation $\mathbf{m}$, ce qui augmente l'angle de précession [1]. Si son amplitude est suffisante pour compenser l'effet des pertes, l'onde est amplifiée au voisinage de la fréquence de résonance.

Dans la géométrie de notre modèle, la résonance ferromagnétique se situe à la pulsation

$$
\omega_{\mathrm{r}}=\gamma\left(B_{0}-\frac{\mu_{0} M_{\mathrm{s}}}{2}\right) .
$$

A cette fréquence, les modes 1 et 2 ont leur énergie concentrée à l'interface des milieux ferrite et semiconducteur (cf. paragraphe 2.2). Il y a donc possibilité d'amplification de l'onde pour ces deux modes.

Avant de passer à une approche purement mathématique, on va approfondir l'étude du mécanisme de l'interaction.

3.2 INTERACTION ENTRE LE FLUX DE PORTEURS ET LE CHAMP ÉLECTRIQUE DE L'ONDE. - La vitesse des électrons dans le semiconducteur s'écrit $\mathbf{V}=\mathbf{v}_{\mathbf{0}}+\mathbf{v}$ où $\mathbf{v}_{0}$ est la composante statique et $\mathbf{v}$ la composante dynamique qui peut s'écrire [3] :

$$
\mathbf{v}=\overrightarrow{\vec{\mu}}_{\mathrm{e}}\left(\mathbf{E}+\mathbf{v}_{0} \times \mathbf{B}+\mathbf{v} \times \mathbf{B}_{0}\right)
$$

avec :

$$
\left.\begin{array}{c}
\overrightarrow{\vec{\mu}}_{\mathrm{e}}=\left[\begin{array}{ccc}
\mu_{\mathrm{t}} & 0 & 0 \\
0 & \mu_{1} & 0 \\
0 & 0 & \mu_{\mathrm{t}}
\end{array}\right] \text { lorsque } \mathbf{v}_{0} \text { est dirigé suivant oy } \\
\mu_{\mathrm{t}}=\frac{V}{E} \\
\mu_{1}=\frac{\partial V}{\partial E}
\end{array}\right\} \text { au point de fonctionnement } .
$$

Soit :

$$
\mathbf{v}=\overrightarrow{\vec{\Gamma}}\left[\mathbf{E}+\mathbf{v}_{\mathbf{0}} \times \mathbf{B}\right]
$$

où $\vec{\Gamma}$ est un tenseur d'ordre 3 (lorsque $\mathbf{B}_{0}$ est dirigé suivant $0 z$, les termes $\Gamma_{13}, \Gamma_{23}, \Gamma_{31}, \Gamma_{32}$ sont nuls).

$\mathbf{E}$ et $\mathbf{v}_{0} \times \mathbf{B}$ sont dirigés suivant $o z$, le terme de vitesse variable $\mathbf{v}$ est donc dirigé aussi suivant $o z$. Il apparaît alors que le champ électrique $\mathbf{E}$ de l'onde exerce une force décélératrice sur les électrons animés d'une vitesse transversale $\mathbf{v}$ (effet Hall). Par contre le champ électrique n'a aucune action sur le terme $\left(v_{0} \times B_{0}\right)$. 
En résumé, le flux d'électrons dans le semiconducteur cède de l'énergie cinétique à l'onde électromagnétique lorsque le champ $\mathbf{E}$ est colinéaire à la vitesse $\mathbf{v}$; il faut donc que la vitesse de phase de l'onde soit sensiblement égale à la vitesse des électrons dans le semiconducteur pour conduire à une amplification de l'onde. Le mécanisme de cette interaction rappelle celui du tube à onde progressive (TOP) où il est possible de décrire le phénomène par la théorie des modes couplés $[4,14]$.

Dans notre cas, il est difficile de définir un coefficient de couplage. Le problème doit donc être traité mathématiquement à partir des résultats généraux du paragraphe 2 .

\section{Etude de la stabilité du système.}

4.1 MÉTHODE UTILISÉE. - Dans les paragraphes précédents, nous avons mis en évidence les possibilités d'amplification des modes de propagation ayant une structure d'onde de surface lorsque la fréquence du signal est voisine de la résonance ferromagnétique $\left(\omega=\omega_{\mathrm{r}}\right)$. Cependant parler d'amplification est prématuré à ce stade de l'étude. En effet, l'existence d'un couplage peut entraîner la mise en oscillation du système, ce qui interdit toute interprétation concernant la vitesse de groupe de l'onde [6]. Ainsi les courbes $k^{\prime \prime}(\omega)$ (Fig. 4) ne permettent de tirer aucune conclusion et une éventuelle amplification ne peut être mise en évidence de cette façon. C'est pourquoi il est nécessaire d'introduire une notion plus générale qui est l'instabilité du système.

Une recherche systématique des conditions d'instabilité a été faite à l'aide du critère de Briggs [6] qui définit deux types d'instabilité dans les systèmes infinis : l'instabilité convective correspondant à une amplification de l'onde et l'instabilité absolue correspondant à une oscillation du système.

Le critère d'instabilité est basé sur l'évolution dans le plan complexe $k$ des racines de l'équation de dispersion $F(\omega, k)=0$, quand la pulsation varie de la valeur réelle $\omega$ à la valeur complexe $\omega-j \infty$. Comme nous allons être amenés à discuter des valeurs complexes de la pulsation, nous noterons dorénavant $\theta=\omega-j X$ avec $(0 \leqslant X \leqslant+\infty)$.

Pour un mode de propagation donné, le système est convectivement instable si la racine correspondante de l'équation de dispersion dans le plan $k$ franchit l'axe réel lorsque $X$ varie de 0 à l'infini. Le système est le siège d'une instabilité absolue quand deux racines provenant l'une du demi-plan $k$ supérieur $\left(k^{\prime \prime}>0\right)$, l'autre du demi plan $k$ inférieur $\left(k^{\prime \prime}<0\right)$ se confondent en une seule racine double pour $X=X_{1}>0$.

4.2 ApPlication DU CRITÈre D'INSTABILITÉ À LA STRUCTURE SEMI-INFINIE YIG-InSb. - Le critère n'est valable que pour les systèmes infinis dans le sens longitudinal. Lorsqu'on limite le système dans cette direction, les résultats obtenus peuvent être modifiés.
Par exemple, une instabilité convective peut devenir absolue par suite de réactions internes (réflexions d'onde) ou disparaître complètement si la longueur du système devient trop faible (notamment un plasma solide ne peut donner d'effet collectif que si la longueur est supérieure à la longueur de Debye [8].

On peut montrer analytiquement que les modes 3 et 4 n'offrent aucune possibilité de stabilité. En effet, il n'existe pas de valeurs réelles de $k_{3}$ ni de $k_{4}$ pour $X>0$ [5].

Les modes 1 et 2 sont étudiés à plusieurs pulsations distinctes notées $\omega_{1}$ à $\omega_{9}$ indiquées sur la figure 7 représentant schématiquement les variations des parties imaginaires $k_{1}^{\prime \prime}$ et $k_{2}^{\prime \prime}$.

Les figures 8 à 14 représentent le lieu des racines $k_{1}$ et $k_{2}$ dans le plan complexe $k=k^{\prime}+j k^{\prime \prime}$ lorsque la partie imaginaire $X$ de la pulsation varie de zéro à l'infini et la partie réelle, constante, prend les valeurs $\omega_{1}$ à $\omega_{9}$. Chaque courbe est paramétrée suivant les valeurs de $X / 2 \pi$ en hertz. Ces résultats correspondent au cas d'une structure YIG-InSb à température ambiante plongée dans une induction magnétique statique de 0,4 T. La vitesse des porteurs dans le semiconducteur est de $10^{5} \mathrm{~m} / \mathrm{s}$.

\section{3 INTERPRÉTATION DES RÉSULTATS.}

4.3.1 Modes 3 et 4. - Un calcul analytique [5] montre que les modes 3 et 4 n'offrent aucune possibilité d'instabilité du système. Ce résultat est relié au fait que pour ces deux modes, la vitesse de phase, comprise entre $1,25 \times 10^{6} \mathrm{~m} / \mathrm{s}$ et $2,5 \times 10^{6} \mathrm{~m} / \mathrm{s}$ reste toujours trop élevée par rapport à la vitesse des porteurs $\left(10^{5} \mathrm{~m} / \mathrm{s}\right)$. Ajoutons que ces modes étant des ondes de volume, nous n'avons à aucun moment la concentration d'énergie à l'interface des deux milieux nécessaire au couplage décrit dans le paragraphe 3.1.

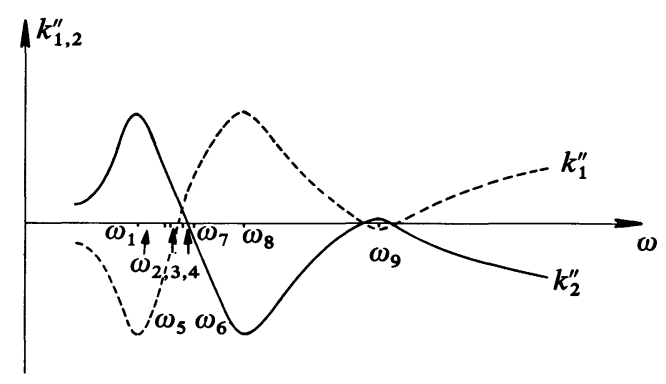

Fig. 7. - Représentation schématique des parties imaginaires $k_{1,2}^{\prime \prime}$ en fonction de la pulsation. Les échelles ne sont pas respectées afin de mieux discerner les positions des différentes pulsations étudiées. Les pulsations $\omega_{2}, \omega_{3}$ et $\omega_{4}$ sont très proches l'une de l'autre et sont telles que $\omega_{2}<\omega_{3}<\omega_{4}$.

[Imaginary parts $k_{1,2}^{\prime \prime}$ schematic mapping versus pulsation $\omega$. Scales are not exact in order to distinguish the different characteristic pulsations. $\omega_{2}, \omega_{3}$ and $\omega_{4}$ are near each others such as $\left.\omega_{2}<\omega_{3}<\omega_{4} \cdot\right]$ 


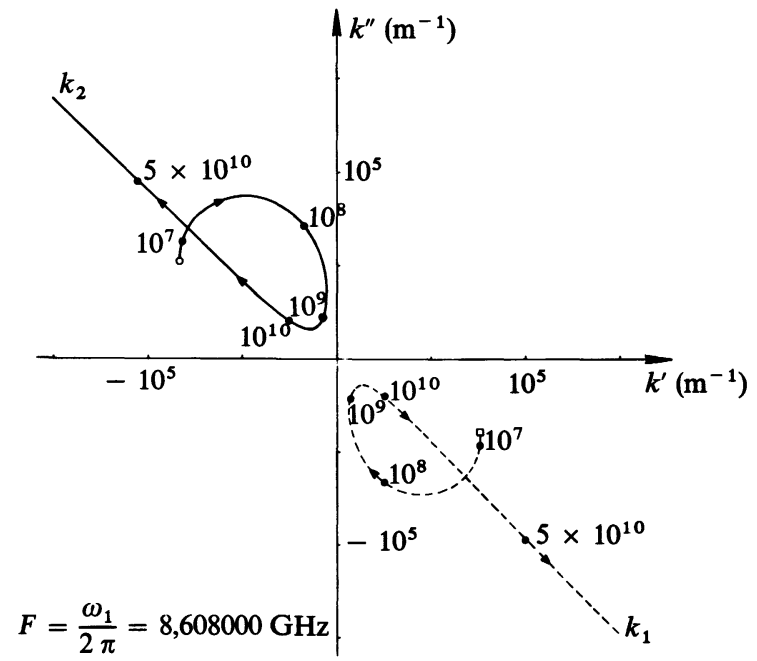

Fig. 8. - Lieu des racines $k_{1}$ et $k_{2}$ pour $\omega=\omega_{1}$ lorsque $X / 2 \pi$ varie de 0 à $5 \times 10^{10} \mathrm{~Hz}$. Aucune des racines ne franchit l'axe $k$ réel, les deux modes sont donc stables à cette fréquence. Remarquons que les racines prennent approximativement la forme $k_{1,2}=\mp \rho \exp j(3 \pi / 4)(\rho$ étant réel) pour les fortes valeurs de $X\left(X / 2 \pi>10^{10} \mathrm{~Hz}\right)$.

[ $k_{1}$ and $k_{2}$ root locus for $\omega=\omega_{1}$ as $X / 2 \pi$ changes from 0 to $5 \times 10^{10} \mathrm{~Hz}$. No root overcomes real axis, both modes are stable at this frequency. Let us remark that roots take nearly the shape $k_{1,2}= \pm \rho \exp j(3 \pi / 4)$ ( $\rho$ is real) for the high values of $X\left(X / 2 \pi>10^{10} \mathrm{~Hz}\right)$.]

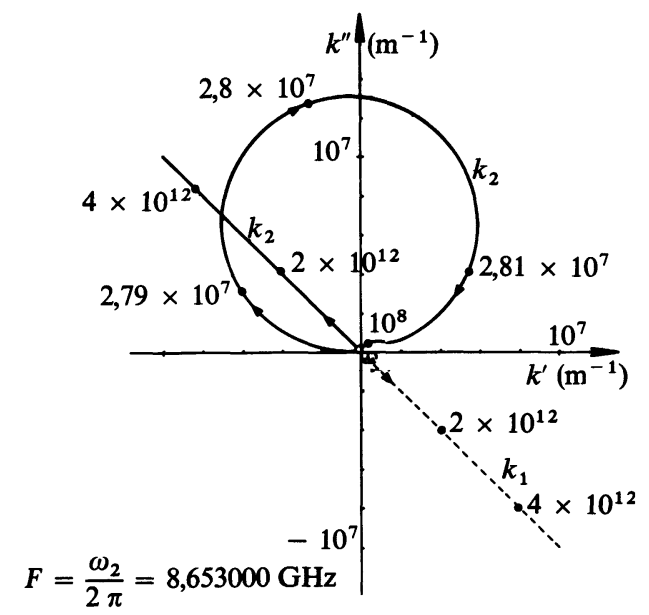

Fig. 9. - Lieu des racines $k_{1}$ et $k_{2}$ pour $\omega=\omega_{2}$ lorsque $X / 2 \pi$ varie de 0 à $5 \times 10^{10} \mathrm{~Hz}$. Les deux modes sont encore stables. Les boucles décrites par les racines sont maintenant très dissymétriques pour les faibles valeurs de $X(X / 2 \pi<$ $10^{10} \mathrm{~Hz}$ ), alors que les deux racines ont comme précédemment une forme symétrique $k_{1,2}=\mp \rho \exp j(3 \pi / 4)$ pour les fortes valeurs de $X\left(X / 2 \pi>10^{10} \mathrm{~Hz}\right)$.

[ $k_{1}$ and $k_{2}$ root locus for $\omega=\omega_{2}$ as $X / 2 \pi$ changes from 0 to $5 \times 10^{10} \mathrm{~Hz}$. Both modes are yet stable. The curves are now asymmetric for the low values of $X\left(X / 2 \pi<10^{10} \mathrm{~Hz}\right)$ and seem as above for high values of $X\left(X / 2 \pi>10^{10} \mathrm{~Hz}\right)$.]

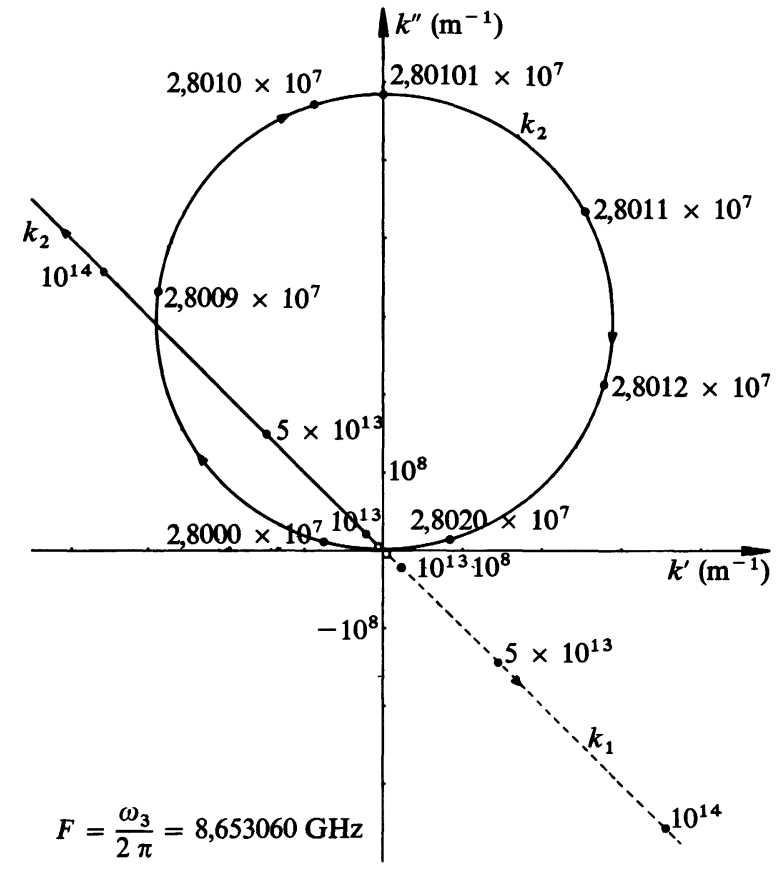

Fig. 10a. - Lieu des racines $k_{1}$ et $k_{2}$ pour $\omega=\omega_{3}$ lorsque $X / 2 \pi$ varie de 0 à $10^{14} \mathrm{~Hz}$. Les deux modes sont stables. Pour les valeurs de $X / 2 \pi$ inférieures à $10^{10} \mathrm{~Hz}, k_{2}$ décrit un cercle de rayon très grand tandis que $k_{1}$ semble rester à son point de départ. Les deux racines prennent ensuite la même forme que précédemment pour les valeurs de $X / 2 \pi$ supérieures à $10^{10} \mathrm{~Hz}$. Dans ce cas, une dilatation d'échelle au voisinage de l'origine des axes s'impose.

$\left[k_{1}\right.$ and $k_{2}$ root locus for $\omega=\omega_{3}$ as $X / 2 \pi$ changes from 0 to $10^{14} \mathrm{~Hz}$. Both modes are stable when $X / 2 \pi<10^{10} \mathrm{~Hz}$. $k_{2}$ describes a large radius circle while $k_{1}$ seems to stay at the starting point. Then both roots take the same shape as above for $X / 2 \pi>10^{10} \mathrm{~Hz}$. In that case a scale expansion is required around the origin point.]

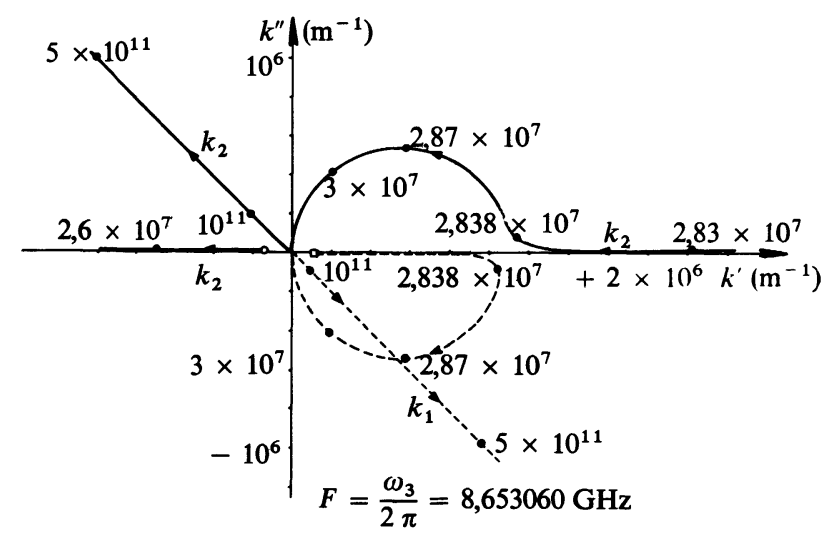

Fig. 10b. - Agrandissement de la figure 10a au voisinage de l'origine. Il doit exister une pulsation réelle proche de $\omega_{3}$ pour laquelle les racines $k_{1}$ et $k_{2}$ se confondent en une seule racine double à l'origine des axes. Le rayon du cercle décrit par $k_{2}$ (cf. Fig. 10a) doit alors tendre vers l'infini.

[Figure 10a enlargement near the origin point. A real pulsation near $\omega_{3}$ from whom $k_{1}$ and $k_{2}$ are coinciding roots must exist near the origin point. $k_{2}$ describes a circle the radius of which tends to infinite.] 
Fig. 11a. - Lieu des racines $k_{1}$ et $k_{2}$ pour $\omega=\omega_{4}$ lorsque $X / 2 \pi$ varie de 0 à $10^{14} \mathrm{~Hz}$. Le départ de la racine $k_{1}$ correspondant à $X=0$ est repéré par le point noté $\square$ alors que celui de la racine $k_{2}$ est repéré par le point noté $O$. Les deux racines franchissent l'axe $k$ réel pour les faibles valeurs de $X$; les deux modes sont donc instables. Le rayon du cercle décrit par $k_{2}$ est très grand et on peut supposer qu'il tend vers l'infini pour une pulsation particulière comprise entre $\omega_{3}$ et $\omega_{4}$. Cette pulsation appelée $\omega_{\mathbf{A}}$ est la pulsation théorique limite inférieure d'une plage d'instabilité (dont la limite supérieure reste à déterminer).

[ $k_{1}$ and $k_{2}$ root locus for $\omega=\omega_{4}$ as $X / 2 \pi$ changes from 0 to $10^{14} \mathrm{~Hz}$. The departure of the $k_{1}$ root corresponding to $X=0$ is marked by the $\square$. Both roots overcome real axis for low $X$ values, both modes are instable. $k_{2}$ describes a circle the radius of which tends to infinite for $\omega_{\mathrm{A}}$ situated between $\omega_{3}$ and $\omega_{4} . \omega_{\mathrm{A}}$ is the theoretical lower limit of an instability band the higher limit of which must be determined.]

Fig. 11b. - Agrandissement de la figure 11a au voisinage de l'origine du lieu des racines $k_{1}$ et $k_{2}$ pour $\omega=\omega_{4}$. Les valeurs des deux racines correspondant à $X=0$ (signes $O$ et $\square$ ) sont peu différentes de celles du cas précédent $\left(\omega=\omega_{3}\right)$. Par contre, les limites de $k_{1}$ et $k_{2}$ lorsque $X$ tend vers l'infini changent de signe par rapport au cas précédent. L'instabilité $\mathrm{du}$ système est convective puisqu'aucune racine double n'apparaît. Par contre, il doit exister une racine double à l'origine des axes pour $\omega=\omega_{\mathrm{A}}$, ce qui révèle une instabilité absolue.

[Figure 11a enlargement around origin point. Both root values corresponding to $X=0$ ( $O$ and $\square$ ) are near than the $\omega=\omega_{3}$ case. On the other hand, as $X$ tends to infinite, the sign of the $k_{1}$ and $k_{2}$ limits changes. There is no coinciding root and the instability is convective. On the other hand a coinciding root must exist at the origin point as $\omega=\omega_{\mathrm{A}}$ and the instability is absolute.]

Fig. 12. - Lieu des racines $k_{1}$ et $k_{2}$ pour les pulsations $\omega_{5}$, $\omega_{6}$ et $\omega_{7}$ lorsque $X / 2 \pi$ varie de 0 à $10^{14} \mathrm{~Hz}$. Les trois courbes sont pratiquement confondues, sauf pour les faibles valeurs de $X\left(X / 2 \pi<10^{7} \mathrm{~Hz}\right)$. Le mode $k_{1}$ devient à nouveau stable pour une pulsation particulière notée $\omega_{\mathbf{B}_{1}}$ comprise entre $\omega_{5}$ et $\omega_{6}$ tandis que le mode $k_{2}$ devient stable pour une pulsation notée $\omega_{\mathbf{B}_{2}}$ comprise entre $\omega_{6}$ et $\omega_{7}$. Ces pulsations sont les limites supérieures des bandes d'instabilité de chaque mode et correspondent au passage par zéro des courbes $k_{1,2}^{\prime \prime}(\omega)$. Nous avons donc d'après la figure $4 \mathrm{c} \omega_{\mathbf{B}_{1}}=\omega_{1}$ et $\omega_{\mathrm{B}_{2}}=\omega_{\mathrm{II}}$.

[ $k_{1}$ and $k_{2}$ root locus for $\omega_{5}, \omega_{6}$ and $\omega_{7}$ pulsations as $X / 2 \pi$ changes from 0 to $10^{14} \mathrm{~Hz}$. The three curves are nearly the same except for lower $X$ values $\left(X / 2 \pi<10^{7} \mathrm{~Hz}\right)$. $k_{1}$ mode is stable for $\omega=\omega_{\mathbf{B}_{1}}$ such as $\omega_{5}<\omega_{\mathbf{B}_{1}}<\omega_{6}$ while $k_{2}$ becomes stable for $\omega=\omega_{\mathrm{B}_{2}}$ such as $\omega_{6}<\omega_{\mathrm{B}_{2}}<\omega_{7}$. These pulsations are the upper limits of each mode instability bands and correspond to $k_{1,2}^{\prime \prime}(\omega)=0$. Figure $4 \mathrm{c}$ gives $\omega_{\mathrm{B}_{1}}=\omega_{\mathrm{I}}$ and $\omega_{\mathrm{B}_{2}}=\omega_{\mathrm{II}} \cdot \mathrm{]}$
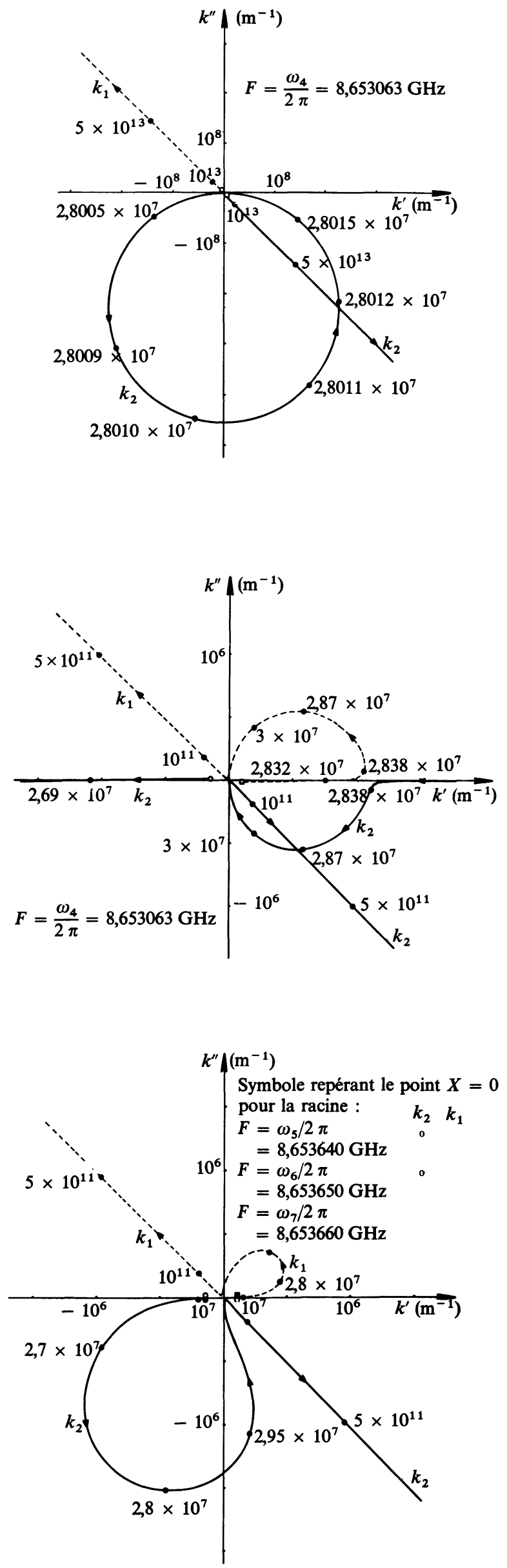


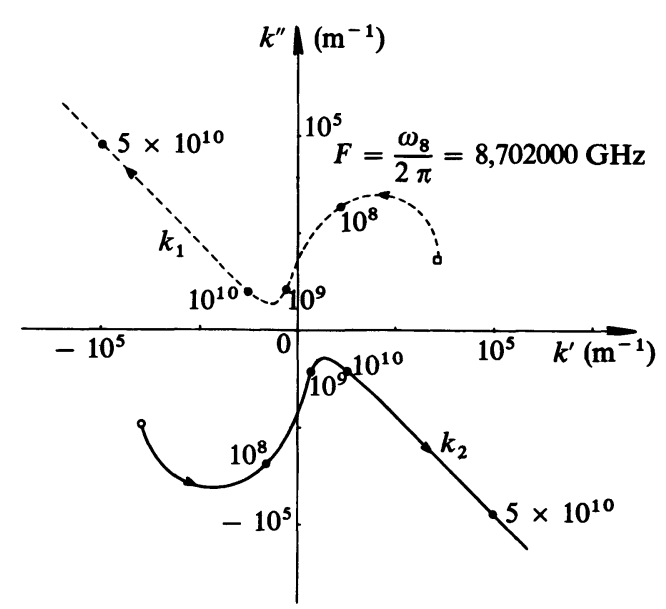

Fig. 13. - Lieu des racines $k_{1}$ et $k_{2}$ pour $\omega=\omega_{8}$ lorsque $X / 2 \pi$ varie de 0 à $5 \times 10^{10} \mathrm{~Hz}$. Aucune des deux racines ne franchit l'axe réel. Les deux modes sont donc stables à cette fréquence.

[ $k_{1}$ and $k_{2}$ root locus for $\omega=\omega_{8}$ as $X / 2 \pi$ changes from 0 to $5 \times 10^{10} \mathrm{~Hz}$. No root overcomes real axis. Both modes are stable.]

4.3.2 Modes 1 et 2. - D'après les résultats du paragraphe 4.2 nous voyons que ces deux modes donnent une instabilité sur une bande de fréquence très étroite centrée sur la fréquence de résonance magnétique. Cette bande d'instabilité est d'environ $590 \mathrm{kHz}$ pour les deux modes.

L'instabilité obtenue est convective sur toute la bande d'instabilité, sauf à la fréquence limite inférieure de cette bande où nous avons une instabilité absolue. Ce résultat est confirmé par le calcul du coefficient d'amplification de l'onde qui tend vers l'infini [5]. L'existence d'une instabilité absolue rend caduques les valeurs de ce coefficient d'amplification. L'interaction de l'onde avec le flux de porteurs à la résonance ferromagnétique ne se limite donc pas à compenser les pertes d'amortissement du mouvement précessionnel de l'aimantation : elle provoque une mise en oscillation du système. Cette oscillation provient du couplage de deux modes dont les vitesses de groupe sont de signe opposé et qui, pour être excités, nécessitent des conditions distinctes : l'un prélève de l'énergie du système, l'autre apporte de l'énergie au système [8]. Il s'agit d'un mode de l'onde de charge d'espace et d'un mode de l'onde électromagnétique. Il ne faut pas confondre ces deux modes avec les modes 1 et 2 qui sont les modes résultant du couplage.

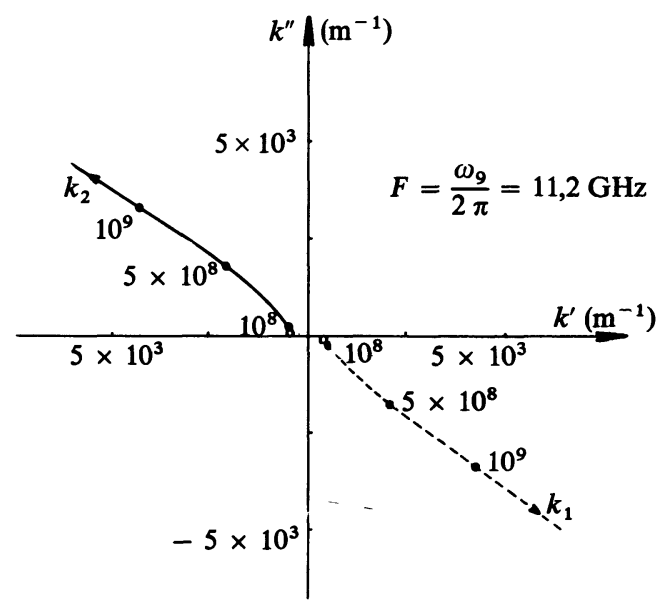

Fig. 14. - Lieu des racines $k_{1}$ et $k_{2}$ lorsque $X / 2 \pi$ varie de 0 à $2 \times 10^{9} \mathrm{~Hz}$. Les deux modes sont stables à cette fréquence. D'autres calculs effectués à des pulsations voisines de $\omega_{9}$ n'ont décelé aucune instabilité pour ces deux modes.

[ $k_{1}$ and $k_{2}$ root locus for $\omega=\omega_{9}$ as $X / 2 \pi$ changes from 0 to $2 \times 10^{9} \mathrm{~Hz}$. Both modes are stable. No instability can be detected around $\omega=\omega_{9}$.]

\section{Conclusion.}

Parmi les modes propres d'une structure semi-infinie ferrite-semiconducteur, seuls les modes TE ont une amplitude suffisante pour interagir avec un flux d'électrons dans le semiconducteur. L'étude de ces modes montre que seulement deux des quatre modes susceptibles de se propager constituent des ondes lentes de surface se propageant dans des directions opposées à la résonance ferromagnétique. Dans ces conditions, une analyse qualitative du mécanisme de l'interaction révèle la possibilité d'échange d'énergie entre l'onde et les porteurs, difficile à exprimer sous la forme d'un couplage entre l'onde électromagnétique et une onde de charge d'espace comme dans un tube à ondes progressives. Nous avons donc été conduits à traiter ce problème par l'application du critère d'instabilité de Briggs [6] aux deux modes lents de surface. Nous avons trouvé que la structure YIG-InSb étudiée était le siège d'une instabilité absolue dans une bande de fréquence très étroite $(\Delta f \simeq 600 \mathrm{kHz})$ centrée sur la fréquence de résonance ferromagnétique $\left(8,65 \mathrm{GHz}\right.$ pour $B_{0}=0,4$ tesla). Cette structure se comporte donc comme un oscillateur de bande très étroite lorsqu'elle a des dimensions infinies. Dans le cas d'une structure de dimensions finies, on peut envisager un comportement d'amplificateur sélectif en bande $\mathbf{X}$. 


\section{Bibliographie}

[1] SCHLÖMANN, E., Amplification of magnetostatic surface waves by interaction with drifting charge carriers in crossed electric and magnetic fields, J. Appl. Phys. 40 (1969) 1422-1424.

[2] Robinson, B. B., Vural, B., Pareckh, J. P., Spinwave/carrier-wave interactions, IEEE Trans. ED 17, no 3 (1970) 224-229.

[3] Bini, M., Filetti, P. L., Millanta, L., Rubino, N., Energetic derivation of the amplification of magnetic waves interacting with a flow of charges in a semiconductor, J.A.P. 47, $\mathrm{n}^{\circ} 7$ (1976) 3209-3218.

[4] Pierce, J. R., Traveling-wave tubes (D. Van Nostrand Company, INC., Princeton New Jersey) 1950.

[5] Rauly, D., Instabilités dans une structure mixteFerrite-semiconducteur propageant une onde de surface, Thèse de 3e cycle, ENSERG-INPG (1982).

[6] BRIGGS, R. J., Electron-stream interaction with plasmas, Research Monograph 29 (The M.I.T. Press, Cambridge, Massachusetts) 1964.

[7] LAX, B., Button, K. J., Microwave ferrites and ferrimagnetics (McGraw-Hill Book Company, INC, New York) 1962.

[8] Steele, M. C., Vural, B., Wave interactions in solid state plasmas (McGraw-Hill advanced Physics Monograph series, New York) 1969.
[9] Thomson-CSF (Domaine de Corbeville), Matériaux ferrites hyperfréquences, Thomson-CSF/DI-DMT/ Paris/12648 (1977).

[10] Caerou, J. C., Application de la méthode des perturbations à l'étude de la conductivité d'un plasma solide anisotrope. Cas de l'Antimoniure d'Indium en bande $X$, Thèse de $3^{\mathrm{e}}$ cycle, U.S.M.G. (1971).

[11] Eshbach, J. R., DAmon, R. W., Surface magnetostatic modes and surface spin waves, Phys. Rev. 118, no 5 (1960) 1208-1210.

[12] Monllor, C., Bouthinon, M., Baribaud, M., Propagation des ondes électromagnétiques dans les milieux présentant une perméabilité et une conductivité tensorielles, Revue Phys. Appl. 13 (1978) 351-365.

[13] RaUly, D., Etude des ondes de surface dans les structures microruban chargées de semiconducteurs et de ferrites, Rapport de D.E.A., ENSERG-INPG (1979).

[14] LOUISELL, W. H., Coupled mode and parametric electronics (John Wiley and Sons, INC, New York, London) 1960. 